$\underline{\text { Supplementary Information }}$

\title{
Poly-phosphocholinated Liposomes Form Stable Superlubrication Vectors
}

Weifeng Lin ${ }^{1}$, Nir Kampf ${ }^{1}$, Ronit Goldberg ${ }^{1}$, Michael J. Driver ${ }^{2}$, Jacob Klein ${ }^{1 *}$

${ }^{1}$ Department of Materials and Interfaces, Weizmann Institute of Science, Rehovot 76100, Israel.

2 Vertellus Biomaterials, Vertellus Specialties UK Ltd., Basingstoke, Hampshire RG25 2PH, U.K.

* Corresponding author:

E-mail address: jacob.klein@weizmann.ac.il 


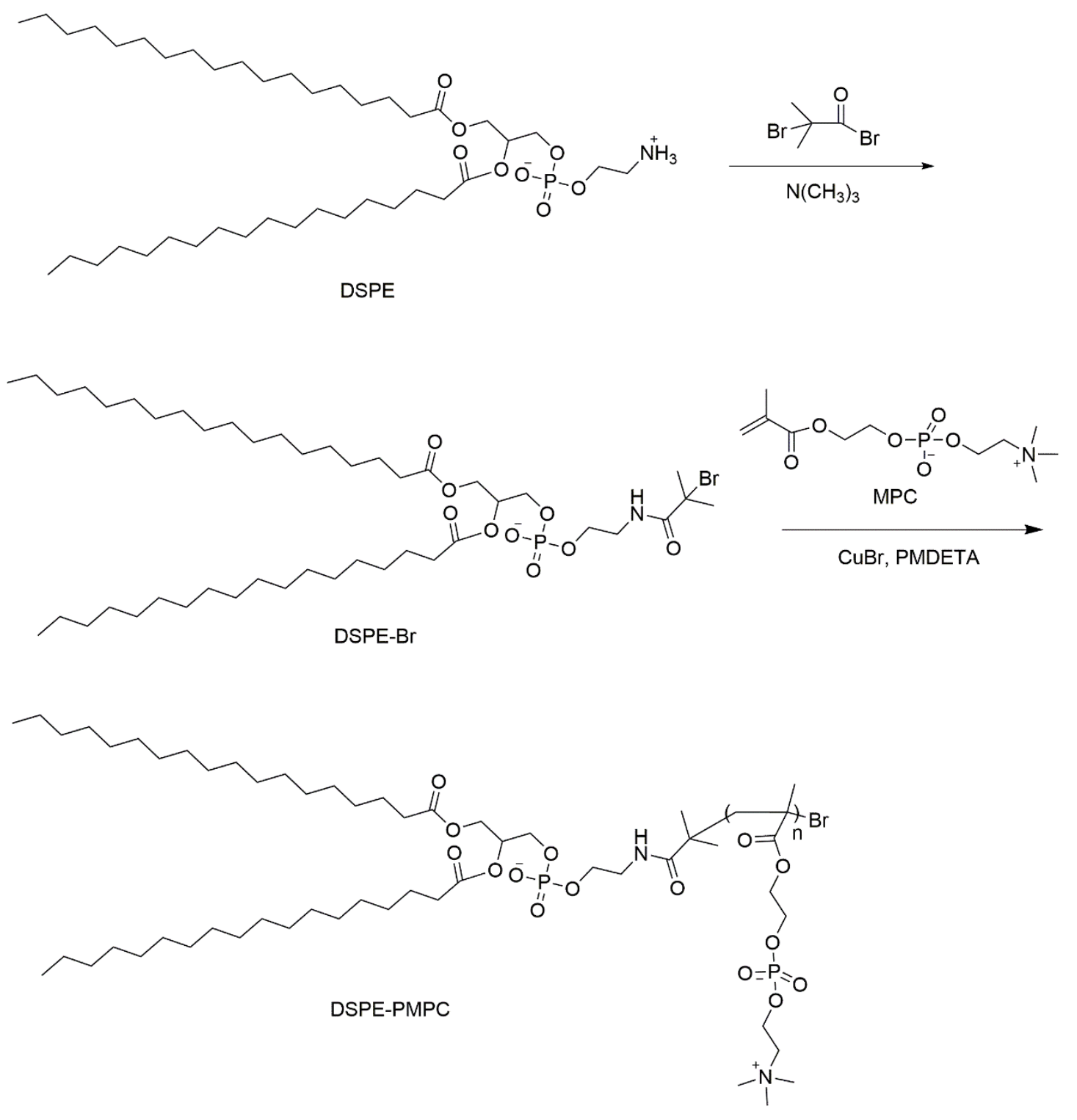

Scheme S1. Schematic illustration of the preparation of DSPE-PMPC polymer via atom transfer radical polymerization, as described in main text.

\section{Control measurements in pure water}

As controls, force profiles were measured between mica surfaces coated with PEGylated and PMPCylated vesicles, prepared in high-purity water with no added salt. These are shown in fig. S1. The curves are a guide to the eye delineating the bulk of the data, and, for the region of onset of interactions ( $D \approx 150-50 \mathrm{~nm}$ ), are reproduced as broken curves in fig. 4a in the main text. Comparison shows that the onset distance for the repulsion was similar both in 0.15 $\mathrm{M}$ salt and in no-added-salt water, revealing the steric rather than electrostatic nature of the interaction, since the latter would be strongly reduced at the high salt concentration. 


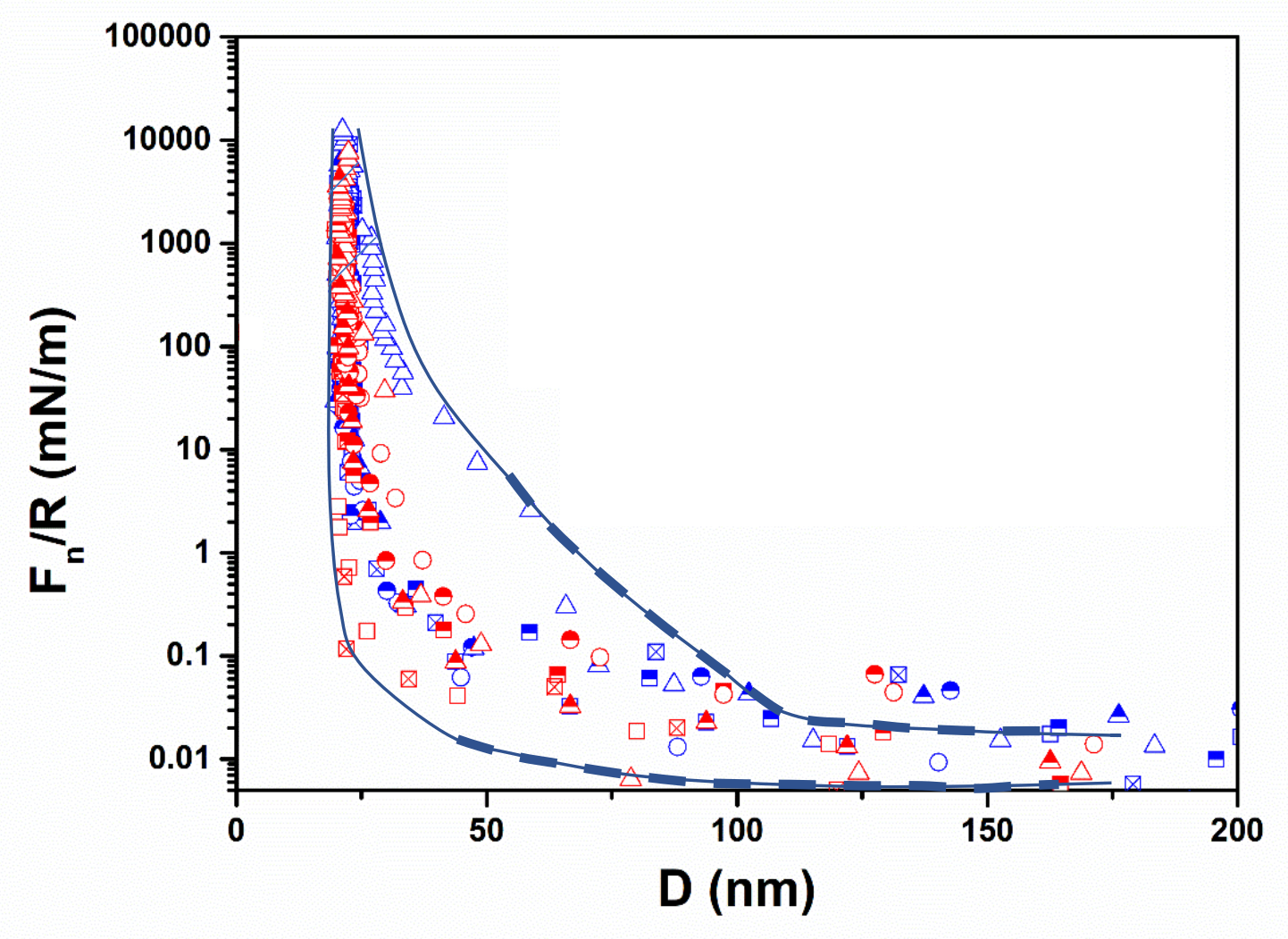

Figure S1. Force distance profiles between mica surfaces coated with PEGylated (blue) and PMPCylated (red) HSPC vesicles, in high purity water with no added salt. The solid curves are a guide to the eye. The onset region from ca. 50 to ca. $150 \mathrm{~nm}$, overlaid by bold broken lines, is reproduced in fig. 4a of the main text. Different shaped symbols correspond to different contact positions with first approaches (empty symbols), second approaches (filled symbols), and retraction profile (crossed symbols). 